\title{
Editoriale
}

\author{
di Francesca Romana Lugeri, Francesco De Pascale, Piero Farabollini
}

La storia della Terra è piena di fenomeni naturali che, per la loro imponenza, hanno influenzato il clima, modificato i paesaggi e la conformazione stessa della superficie del pianeta. Solitamente, essi sono un continuum di eventi che ricorrono periodicamente. I terremoti e le eruzioni vulcaniche, e i maremoti che a volte ne conseguono, non sono che la manifestazione superficiale dei movimenti delle placche tettoniche e della dinamica interna del pianeta, così come le alluvioni, gli uragani e i tornado non sono che gli effetti di dinamiche meteorologiche e - su scala globale - climatiche, mentre le frane sono la risposta agli agenti che tendono a modificare i rilievi. Gli stessi impatti tra corpi celesti rispondono alla dinamica che regola l'universo. È l'interferenza con le attività antropiche che trasforma gli eventi naturali in disastri. E maggiore è l'aumento della popolazione umana sulla Terra, con la ricerca continua di nuovi spazi che porta l'uomo a vivere e costruire in zone a rischio, maggiore è la possibilità che le catastrofi avvengano.

In particolare, l'Italia è un paese che, per caratteristiche fisiografiche ed esposizione ai rischi idrogeologico, sismico e vulcanico, ben rappresenta la coesistenza, talvolta la contrapposizione di due coscienze: quella della responsabilità e quella del fatalismo. Il confronto è reso più complesso dalla presenza di interessi e speculazioni.

Cosa è possibile fare per lenire, se non evitare, gli effetti dannosi dei fenomeni naturali? Una valida impostazione per ottenere delle soluzioni è basata sull'investimento nella conoscenza, finalizzata alla prevenzione, utilizzando le conquiste scientifiche e tecnologiche per la diffusione e il miglioramento delle reti di monitoraggio ed applicando efficacemente criteri di salvaguardia ambientale e di sviluppo sicuro e sostenibile. È l'attuale società umana disponibile a questo? Non si può non essere pessimisti, viste l'inadeguatezza delle risorse finanziarie e la scarsa fluidità della burocrazia, a fronte della frequente costruzione di opere, spesso inutili se non dannose.

Inoltre, si continua a registrare una continua e sconcertante carenza di chiarezza sul concetto di rischio. Largamente utilizzato quando si parla di catastrofi, l'uso del termine non è, tuttavia, chiaro ed univoco. Per evitare ambiguità, è opportuno esprimere il rischio in termini analitici attraverso una formula che lega pericolosità, vulnerabilità e valore esposto. Il rischio è il prodotto della probabilità di accadi- 
mento di un evento per le dimensioni del danno atteso ed esprime, dunque, le conseguenze di un particolare evento dannoso: tale espressione implica il ruolo della responsabilità civile e sociale. L'esposizione ai fenomeni naturali comporta spesso il verificarsi di conseguenze tali da sconvolgere, anche in modo permanente, l'esistenza degli individui, delle comunità attraversate da eventi estremi di particolare intensità. L'entità dei danni e delle perdite è in funzione dell'assetto del territorio colpito: posizione geografica, strutture e infrastrutture presenti, densità della popolazione. Pertanto, è necessario utilizzare un approccio integrato nello studio e nella prevenzione dei disastri: dai sistemi di allerta alla definizione/costruzione del rischio, dalla vulnerabilità sociale alla resilienza territoriale, dalla valutazione alla gestione, dalla comunicazione alla percezione del rischio e alla progettazione partecipata (approccio bottom-up invece che top-down), incrociando altresì gli aspetti storici, economici, geografici, psicologici e socio-culturali (Carnelli e Ventura, 2015; Carnelli, Forino, 2017).

Scriveva Khalil Gibran: "la nostra casa è il nostro corpo più grande". La casa, in quasi tutte le culture, assume un significato esistenziale: un insieme di case individua la presenza di una forma di società. Un disastro, insieme alle case, spazza via i punti di riferimento sociali ed emotivi degli individui. Le reazioni psicologiche alla catastrofe sono finalmente divenute oggetto di studi specifici, nel nostro paese, dal terremoto che nel 1997 ha colpito Umbria e Marche. Soltanto in seguito ad esso sono nate organizzazioni per la Psicologia dell'Emergenza (De Felice e Colaninno, 2003; Barbato et al., 2006), ma ancora ad oggi la società italiana non ha sviluppato una cultura finalizzata alla prevenzione efficace dei rischi legati ad eventi naturali estremi.

L'assenza di una "cultura del rischio" si avverte ancora nel campo della comunicazione, che abbiamo il dovere di far diventare potente strumento per diffondere conoscenza, patrimonio prezioso in situazioni di emergenza. Ed è nostro - comune - dovere, soffermarci sul particolare significato di questo Special Issue, che vede, tra i curatori e gli autori, il Commissario Straordinario alla Ricostruzione postsisma, Piero Farabollini, docente di Geomorfologia all'Università di Camerino, nominato dal governo Conte il 5 ottobre 2018 per favorire un approccio tecnico alla ricostruzione. La profonda conoscenza del territorio colpito dal sisma è alla base della fase di gestione commissariale, articolata su rigorose analisi scientifiche e su procedure tecniche sviluppate ad hoc, come illustrate nel contributo pubblicato.

I disastri sono la risultante dell'interazione tra fattori naturali e azione dell'uomo. Spesso, un insieme di cause concomitanti determinano effetti di entità imprevedibile. «Non ci sono nemmeno catastrofi "puramente" naturali. In esse è sempre implicato anche l'agire - o il non-agire - umano» (Beck, 2000).

I tempi "geologici" sono molto diversi da quelli "umani", essendo la scala dei primi basata su decine e centinaia di migliaia o addirittura milioni di anni, mentre $\mathrm{i}$ secondi non superano le decine, centinaia o, al massimo, le migliaia di anni. Ciò non esclude, però, che certi fenomeni si siano verificati e che le cause scatenanti siano tuttora esistenti e pronte, prima o poi, a scaricare la loro energia distruttiva. 
Per questo motivo, ai fini di ridurre al minimo la vulnerabilità sociale a questa tipologia di eventi naturali estremi, è fondamentale potenziare gli interventi non strutturali di mitigazione del rischio come le azioni di prevenzione e di preparazione delle comunità ai disastri, le attività psico-sociali in emergenza e la comunicazione del rischio.

Con questo volume, pertanto, non si vogliono offrire delle soluzioni, ma degli strumenti per affrontare con adeguata competenza la complessità della comunicazione e divulgazione del rischio. Tutti gli scritti proposti sono dedicati alle varie possibili declinazioni dei temi relativi al rapporto tra rischio e società. La molteplicità dei punti di vista contribuisce alla completezza della raccolta, grazie al consistente insieme di esperienze, idee, proposte.

Nello specifico, Di Bucci analizza pericolosità e rischio in termini di risposta sociale, attraverso gli strumenti dell'analisi comportamentale: tema questo emerso solo di recente nel campo delle attività di protezione civile.

Parallelamente, l'articolo di Fermani e Carreri è concentrato sulle percezioni a livello individuale nei contesti di rischio, analisi molto importante per una migliore prevenzione.

Anche lo studio di Antronico, Coscarelli, De Pascale e Gullà esamina la percezione sociale del rischio, utilizzando metodi qualitativi, in relazione all'evento di Maierato (VV), colpito da un fenomeno franoso nel 2010.

Nell'articolo di Piangiamore vengono passate in rassegna le esperienze realizzate in ambito educativo, finalizzate a coinvolgere l'intera comunità e, soprattutto, la scuola, in attività continuative di diffusione della cultura della prevenzione.

Lugeri concentra l'attenzione sull'importanza della comunicazione, proponendo nuove strategie per la condivisione della conoscenza scientifica, utilizzando come vettori d'informazione argomenti più accessibili e graditi al pubblico.

Il lavoro di Dattilo si focalizza sul collegamento tra il concetto di comunicazione e quello di azione e sull'aspetto semiotico della problematica, con implicazioni profonde sul rischio antropologico permanente.

Un'originale formula caratterizza la proposta di Farabollini, un contributo di taglio esplicitamente divulgativo e 'tecnico', che analizza dalla prospettiva privilegiata del Commissario Straordinario le procedure di ricostruzione post-sisma, chiarendone complessità e difficoltà procedurali, in una sorta di alfabeto, strumento primario per la decodifica e la comprensione.

Dunque, l'intento principale, il comune denominatore di tutti i contributi raccolti in questo volume riguarda l'importanza di comunicare e di essere compresi. Solo attraverso uno scambio osmotico di conoscenza si può sperare in un futuro che veda il nostro Paese, così bello e al tempo stesso così fragile, sviluppare realtà di prevenzione efficace, di resilienza, di sostenibilità e di benessere condiviso.

\section{Riferimenti bibliografici}

Barbato R., Puliatti M., Micucci M., (2006) Psicologia dell'Emergenza. Manuale di intervento sulle crisi da eventi catastrofici, Edup, Roma 
Beck U., (1986) Risikogesellschaft: Auf dem Weg in eine andere Moderne, Suhrkamp, Verlag, Frankfurt am Main; trad. it. La Società del rischio. Verso una seconda modernità, Carocci, Roma, 2000

Carnelli F., Forino G., (2017) Gestire un terremoto in Italia: breve prontuario di emergenza sismica, Lo stato delle cose. Geografie e storie del doposisma, -$\mathrm{http} / /$ www.lostatodellecose.com/scritture/gestire-un-terremoto-in-italia-breveprontuario-demergenza-sismica-fabio-carnelli-e-giuseppe-forino/ (ultimo accesso: $22 / 07 / 2019)$

Carnelli F., Ventura S., (2015) (a cura di) Oltre il Rischio Sismico. Valutare, Comunicare e decidere Oggi, Carocci, Roma

De Felice F., Colaninno C., (2003) Psicologia dell'Emergenza, FrancoAngeli, Milano 